\title{
COMMON FIXED POINTS OF A PAIR OF NON-EXPANSIVE MAPPINGS WITH APPLICATIONS TO CONVEX FEASIBILITY PROBLEMS
}

\author{
XIAOLONG QIN*, SUN YOUNG CHO \\ Department of Mathematics, Gyeongsang National University, Chinju 660-701, Korea \\ e-mails:qxlxajh@163.com,ooly61@yahoo.co.kr
}

\author{
and HAIYUN ZHOU
} Department of Mathematics, Shijiazhuang Mechanical Engineering College, Shijiazhuang 050003, China
e-mail:witman66@yahoo.com.cn

(Received 17 November 2008; accepted 25 August 2009; first published online 25 November 2009)

\begin{abstract}
Let $C$ be a non-empty closed convex subset of a reflexive and strictly convex Banach space $E$ which also has a weakly continuous duality map $J_{\varphi}(x)$ with the gauge $\varphi$. Let $S$ and $T$ be non-expansive mappings from $C$ into itself such that $F=F(S) \cap F(T) \neq \emptyset$. Let $\left\{\alpha_{n}\right\}$ and $\left\{\beta_{n}\right\}$ be sequences in $(0,1)$. Let $\left\{x_{n}\right\}$ be a sequence defined by

$$
\left\{\begin{array}{l}
x_{0} \in C, \\
y_{n}=\beta_{n} S x_{n}+\left(1-\beta_{n}\right) T x_{n}, \\
x_{n+1}=\alpha_{n} u+\left(1-\alpha_{n}\right) y_{n}, \quad n \geq 0,
\end{array}\right.
$$

where $u \in C$ is a given point. Assume that the following restrictions imposed on the control sequences are satisfied:

(a) $\sum_{n=0}^{\infty} \alpha_{n}=\infty, \lim _{n \rightarrow \infty} \alpha_{n}=0$;

(b) $\sum_{n=0}^{\infty}\left|\alpha_{n+1}-\alpha_{n}\right|<\infty, \sum_{n=0}^{\infty}\left|\beta_{n+1}-\beta_{n}\right|<\infty$;

(c) $\lim _{n \rightarrow \infty} \beta_{n}=\beta \in(0,1)$.

Then the sequence $\left\{x_{n}\right\}$ converges strongly to $x^{*} \in F$, where $x^{*}=Q(u)$ and $Q: C \rightarrow F$ is the unique sunny non-expansive retraction from $C$ onto $F$.
\end{abstract}

2000 AMS Subject Classification. 47H09; 47H10; 47J25.

1. Introduction and preliminaries. Throughout this paper, we always assume that $E$ is a real Banach space. Let $E^{*}$ be the dual space of $E$. Let $\varphi:[0, \infty]:=R^{+} \rightarrow R^{+}$ be a continuous and strictly increasing function such that $\varphi(0)=0$ and $\varphi(t) \rightarrow \infty$ as $t \rightarrow \infty$. This function $\varphi$ is called a gauge function. The duality mapping $J_{\varphi}: E \rightarrow E^{*}$ associated with a gauge function $\varphi$ is defined by

$$
J_{\varphi}(x)=\left\{f^{*} \in E^{*}:\left\langle x, f^{*}\right\rangle=\|x\| \varphi(\|x\|), \quad\left\|f^{*}\right\|=\varphi(\|x\|)\right\}, \quad \forall x \in E,
$$

where $\langle\cdot, \cdot\rangle$ denotes the generalized duality pairing. In the case that $\varphi(t)=t$, we write $J$ for $J_{\varphi}$ and call $J$ the normalized duality mapping.

*Corresponding author. 
Following Browder [2], we say that a Banach space $E$ has a weakly continuous duality mapping if there exists a gauge $\varphi$ for which the duality mapping $J_{\varphi}(x)$ is singlevalued and weak-to-weak* sequentially continuous (i.e., if $\left\{x_{n}\right\}$ is a sequence in $E$ weakly convergent to a point $x$, then the sequence $J_{\varphi}\left(x_{n}\right)$ converges weakly* to $J_{\varphi} x$ ). It is known that $l^{p}$ has a weakly continuous duality mapping with a gauge function $\varphi(t)=t^{p-1}$ for all $1<p<\infty$. Set

$$
\Phi(t)=\int_{0}^{t} \varphi(\tau) d \tau, \quad \forall t \geq 0,
$$

then

$$
J_{\varphi}(x)=\partial \Phi(\|x\|), \quad \forall x \in E,
$$

where $\partial$ denotes the sub-differential in the sense of convex analysis.

The norm of $E$ is said to be Gâteaux differentiable (and $E$ is said to be smooth) if

$$
\lim _{t \rightarrow 0} \frac{\|x+t y\|-\|x\|}{t}
$$

exists for each $x, y$ in its unit sphere $U_{E}=\{x \in E:\|x\|=1\}$. It is said to be uniformly Fréchet differentiable (and $E$ is said to be uniformly smooth) if the limit is attained uniformly for $(x, y) \in U_{E} \times U_{E}$.

A Banach space $E$ is said to strictly convex if and only if

$$
\|x\|=\|y\|=\|(1-\lambda) x+\lambda y\|
$$

for all $x, y \in E$ and $0<\lambda<1$ implies that $x=y$. $E$ is said to uniformly convex if, for any $\epsilon \in(0,2]$, there exists $\delta>0$ such that, for any $x, y \in U$,

$$
\|x-y\| \geq \epsilon \text { implies }\left\|\frac{x+y}{2}\right\| \leq 1-\delta .
$$

It is known that a uniformly convex Banach space is reflexive and strictly convex.

Let $C$ be a non-empty closed convex subset of a real Banach space $E$ and $T: C \rightarrow$ $C$ a nonlinear mapping. A point $x \in C$ is a fixed point of $T$ provided that $T x=x$. In this paper, we use $F(T)$ to denote the set of fixed points of $T$.

Recall that a mapping $T$ is non-expansive if

$$
\|T x-T y\| \leq\|x-y\|, \quad \forall x, y \in C .
$$

One classical way to study non-expansive mappings is to use contractions to approximate a non-expansive mapping ([1, 23, 31]). More precisely, take $t \in(0,1)$ and define a contraction $T_{t}: C \rightarrow C$ by

$$
T_{t} x=t u+(1-t) T x, \quad \forall x \in C,
$$

where $u \in C$ is a fixed point. Banach's contraction mapping principle guarantees that $T_{t}$ has a unique fixed point $x_{t}$ in $C$. That is,

$$
x_{t}=t u+(1-t) T x_{t} .
$$

It is unclear, in general, what is the behaviour of $x_{t}$ as $t \rightarrow 0$, even if $T$ has a fixed point. However, in the case of $T$ having a fixed point, Browder [1] proved that if $E$ is a Hilbert 
space, then $x_{t}$ converges strongly to a fixed point of $T$. Reich [23] extended Broweder's result to the setting of Banach spaces and proved that if $E$ is a uniformly smooth Banach space, then $x_{t}$ converges strongly to a fixed point of $T$ and the limit defines the (unique) sunny non-expansive retraction from $C$ onto $F(T) . \mathrm{Xu}$ [32] proved that Broweder's results still hold in reflexive Banach spaces which have a weakly continuous duality mapping; see [32] for more details.

Recall that if $C$ and $D$ are non-empty subsets of a Banach space $E$ such that $C$ is non-empty closed convex and $D \subset C$, then a map $Q: C \rightarrow D$ is called a retraction from $C$ onto $D$ provided $Q(x)=x$ for all $x \in D$. A retraction $Q: C \rightarrow D$ is sunny provided $Q(Q(x)+t(x-Q(x)))=Q(x)$ for all $x \in C$ and $t \geq 0$ whenever $Q(x)+t(x-Q(x)) \in C$. A sunny non-expansive retraction is a sunny retraction which is also non-expansive. Sunny non-expansive retractions are characterized as follows $[\mathbf{9}, \mathbf{2 3}]$ :

If $E$ is a smooth Banach space, then $Q: C \rightarrow D$ is a sunny non-expansive retraction if and only if there holds the inequality

$$
\langle x-Q x, J(y-Q x)\rangle \leq 0, \quad \forall x \in C, \quad y \in D .
$$

Reich [23] showed that if $E$ is uniformly smooth and $D$ is the fixed point set of a non-expansive mapping from $C$ into itself, then there is a unique sunny non-expansive retraction from $C$ onto $D$ and it can be constructed as follows.

THEOREM R. Let E be a uniformly smooth Banach space and let $T: C \rightarrow C$ be a nonexpansive mapping with a fixed point. For each fixed $u \in C$ and every $t \in(0,1)$, the unique fixed point $x_{t} \in C$ of the contraction $C \ni x \mapsto t u+(1-t) T x$ converges strongly as $t \rightarrow 0$ to a fixed point of $T$. Define $Q: C \rightarrow D$ by $Q u=s-\lim _{t \rightarrow 0} x_{t}$. Then $Q$ is the unique sunny non-expansive retract from $C$ onto $D$; that is, $Q$ satisfies the property:

$$
\langle u-Q u, J(y-Q u)\rangle \leq 0, \quad \forall u \in C, y \in D .
$$

If $E$ is a reflexive Banach space which has a weakly contnuous duality map, then $Q: C \rightarrow D$ is a sunny non-expansive retraction if and only if there holds the inequality

$$
\left\langle x-Q x, J_{\varphi}(y-Q x)\right\rangle \leq 0, \quad \forall x \in C, \quad y \in D .
$$

In 2006, Xu [32] obtained an analogue of Theorem $R$ in a reflexive Banach space. To be more precise, he proved the following result.

THEOREM X. Let $E$ be a reflexive Banach space and has a weakly continuous duality map $J_{\varphi}(x)$ with gauge $\varphi$. Let $C$ be closed convex subset of $E$ and let $T: C \rightarrow C$ be a non-expansive mapping. Fix $u \in C$ and $t \in(0,1)$. Let $x_{t} \in C$ be the unique solution in $C$ to the equation (1.2). Then $T$ has a fixed point if and only if $\left\{x_{t}\right\}$ remains bounded as $t \rightarrow 0^{+}$, and in this case, $\left\{x_{t}\right\}$ converges as $t \rightarrow 0^{+}$strongly to a fixed point of $T$.

Under the condition of Theorem $X$, define a mapping $Q: C \rightarrow F(T)$ by

$$
Q(u):=\lim _{t \rightarrow 0} x_{t}, \quad \forall u \in C .
$$

From $\mathrm{Xu}$ [32, Theorem 3.2], we know that $Q$ is the sunny non-expansive retraction from $C$ onto $F(T)$.

Two iteration processes are often used to approximate a fixed point of a nonexpansive mapping $T$. 
The first one is introduced by Halpern [10]. The Halpern's iterative process generates a sequence $\left\{x_{n}\right\}$ in the following manner:

$$
\left\{\begin{array}{l}
x_{1} \in C, \quad \text { chosen arbitrarily, } \\
x_{n+1}=\alpha_{n} u+\left(1-\alpha_{n}\right) T x_{n}, \quad \forall n \geq 1
\end{array}\right.
$$

where $u \in C$ is a given point and the sequence $\left\{\alpha_{n}\right\}$ is in the interval $(0,1)$. Halpern proved that the strong convergence of $\left\{x_{n}\right\}$ to a fixed point of $T$ in a real Hilbert space provided that $\alpha_{n}=n^{-\theta}$, where $\theta \in(0,1)$.

In 1977, Lions [15] improved the result of Halpern [10], still in Hilbert spaces, by proving the strong convergence of $\left\{x_{n}\right\}$ to a fixed point of $T$ where the real sequence $\left\{\alpha_{n}\right\}$ satisfies the following conditions:

$$
\text { (C1): } \lim _{n \rightarrow \infty} \alpha_{n}=0,(C 2): \sum_{n=1}^{\infty} \alpha_{n}=\infty \text { and }(C 3): \lim _{n \rightarrow \infty} \frac{\alpha_{n+1}-\alpha_{n}}{\alpha_{n+1}^{2}}=0 .
$$

It was observed that both Halperns and Lions conditions on the real sequence $\left\{\alpha_{n}\right\}$ excluded the canonical choice $\left\{\alpha_{n}\right\}=\frac{1}{n+1}$. This was overcome in 1992 by Wittmann [28], who proved, still in Hilbert spaces, the strong convergence of $\left\{x_{n}\right\}$ to a fixed point of $T$ if $\left\{\alpha_{n}\right\}$ satisfies the following conditions:

$$
\text { (C1) : } \lim _{n \rightarrow \infty} \alpha_{n}=0,(C 2): \sum_{n=1}^{\infty} \alpha_{n}=\infty \text { and }(C 4): \sum_{n=1}^{\infty}\left|\alpha_{n+1}-\alpha_{n}\right|<\infty \text {. }
$$

In 2002, Xu [30] (see also [29]) further improved the result of Lion's. To be more precise, he weakened the condition $(C 3)$ by removing the square in the denominator so that the canonical choice of $\left\{\alpha_{n}\right\}=\frac{1}{n+1}$ is possible.

The second one is the normal Mann's iterative process [16] which generates a sequence $\left\{x_{n}\right\}$ in the following manner:

$$
\left\{\begin{array}{l}
x_{1} \in C, \quad \text { chosen arbitrarily, } \\
x_{n+1}=\left(1-\alpha_{n}\right) x_{n}+\alpha_{n} T x_{n}, \quad \forall n \geq 1,
\end{array}\right.
$$

where the sequence $\left\{\alpha_{n}\right\}$ is in the interval $(0,1)$.

If $T$ is a non-expansive mapping with a fixed point and the control sequence $\left\{\alpha_{n}\right\}$ is chosen so that $\sum_{n=0}^{\infty} \alpha_{n}\left(1-\alpha_{n}\right)=\infty$, then the sequence $\left\{x_{n}\right\}$ generated by normal Mann's iterative process (1.2) converges weakly to a fixed point of $T$ (this is also valid in a uniformly convex Banach space with the Fréchet differentiable norm [22]). In an infinite-dimensional Hilbert space, the normal Mann's iteration algorithm has only weak convergence. Therefore, many authors try to modify the normal Mann's iteration process to have strong convergence for non-expansive mappings (see [5-7, 11, 17-21, $\mathbf{2 4}, \mathbf{3 3}, \mathbf{3 4}$ ] and the references therein).

In 2005, Kim and Xu [11] modified normal Mann's iterative method for a single non-expansive mappings in a uniformly smooth Banach space. To be more precise, they proved the following result.

THEOREM KX. Let $C$ be a closed convex subset of a uniformly smooth Banach space $E$ and let $T: C \rightarrow C$ be a non-expansive mapping such that $F(T) \neq \emptyset$. Given a point $u \in C$ and given sequences $\left\{\alpha_{n}\right\}$ and $\left\{\beta_{n}\right\}$ in $(0,1)$, the following conditions are satisfied:

(1) $\alpha_{n} \rightarrow 0, \beta_{n} \rightarrow 0$ 
(2) $\sum_{n=0}^{\infty} \alpha_{n}=\infty, \sum_{n=0}^{\infty} \beta_{n}=\infty$;

(3) $\sum_{n=0}^{\infty}\left|\alpha_{n+1}-\alpha_{n}\right|<\infty, \quad \sum_{n=0}^{\infty}\left|\beta_{n+1}-\beta_{n}\right|<\infty$.

Define a sequence $\left\{x_{n}\right\}$ in $C$ by

$$
\left\{\begin{array}{l}
x_{0}=x \in C \quad \text { chosen arbitrarily, } \\
y_{n}=\beta_{n} x_{n}+\left(1-\beta_{n}\right) T x_{n}, \\
x_{n+1}=\alpha_{n} u+\left(1-\alpha_{n}\right) y_{n}, \quad \forall n \geq 0 .
\end{array}\right.
$$

Then $\left\{x_{n}\right\}$ strongly converges to a fixed point of $T$.

Very recently, Qin, Su and Wu [21] further improved Kim and Xu [11]'s results by considering a pair of non-expansive mappings in a uniformly smooth Banach space. More precisely, they obtained the following result.

THEOREM QSW. Let $C$ be a closed convex subset of a uniformly smooth Banach space $E$ and $f: C \rightarrow C$ a contractive mapping. Let $S: C \rightarrow C$ and $T: C \rightarrow C$ be a pair of non-expansive mappings such that $F(S T)=F(S) \bigcap F(T) \neq \emptyset$. The initial guess $x_{0} \in C$ is chosen arbitrarily and given sequences $\left\{\alpha_{n}\right\},\left\{\beta_{n}\right\}$ and $\left\{\gamma_{n}\right\}$ in $[0,1]$, the following conditions are satisfied

(a) $\alpha_{n} \rightarrow 0, \sum_{n=0}^{\infty} \alpha_{n}=\infty$ and $\sum_{n=0}^{\infty}\left|\alpha_{n+1}-\alpha_{n}\right|<\infty$;

(b) $\beta_{n} \rightarrow 0, \gamma_{n} \rightarrow 0, \sum_{n=0}^{\infty}\left|\beta_{n+1}-\beta_{n}\right|<\infty$ and $\sum_{n=0}^{\infty}\left|\gamma_{n+1}-\gamma_{n}\right|<\infty$.

Let $\left\{x_{n}\right\}$ be the sequence defined by

$$
\left\{\begin{array}{l}
z_{n}=\gamma_{n} x_{n}+\left(1-\gamma_{n}\right) T x_{n}, \\
y_{n}=\beta_{n} x_{n}+\left(1-\beta_{n}\right) S z_{n}, \\
x_{n+1}=\alpha_{n} f\left(x_{n}\right)+\left(1-\alpha_{n}\right) y_{n}, \quad n \geq 0 .
\end{array}\right.
$$

Then $\left\{x_{n}\right\}_{n=1}^{\infty}$ converges strongly to some common fixed point $p \in F\left(T_{1}\right) \cap F\left(T_{2}\right)$

Motivated by Kimura, Takahashi and Toyoda [12], Kim and Xu [11] and Qin et al. [21], we consider the following iterative method

$$
\left\{\begin{array}{l}
x_{0}=x \in C \quad \text { chosen arbitrarily, } \\
y_{n}=\beta_{n} S x_{n}+\left(1-\beta_{n}\right) T x_{n}, \quad \forall n \geq 0 \\
x_{n+1}=\alpha_{n} u+\left(1-\alpha_{n}\right) y_{n}, \quad \forall n
\end{array}\right.
$$

where $\left\{\alpha_{n}\right\},\left\{\beta_{n}\right\}$ are sequences in $(0,1)$ and $S$ and $T$ are non-expansive mappings.

We remark that our iterative method $(\Upsilon)$ is more general.

(1) If $S=I$, the identity mapping, then the above iterative process reduces to the $(M M I P)$.

(2) If $S=T$, then the above iterative process collapses to (HIP).

In this paper, we study the above iterative process for a pair of non-expansive mappings. Strong convergence theorems are established in a reflexive Banach space. As applications, we consider the convex feasibility problem (CFP) of finding a point in the non-empty intersection $C_{i=1}^{N}$, where $N \geq 1$ is an integer and each $C_{i}$ is assumed to be the fixed point set of a non-expansive mapping $T_{i}: C \rightarrow C$, where $C$ is a non-empty closed and convex subset of $E$.

In order to prove our main results, we need the following lemmas.

The first part of the next lemma is an immediate consequence of the sub-differential inequality and the proof of the second part can be found in [14]. 
LEMMA 1.1. Assume that a Banach space E has a weakly continuous duality mapping $J_{\varphi}$ with a gauge $\varphi$.

(i) For all $x, y \in E$, the following inequality holds:

$$
\Phi(\|x+y\|) \leq \Phi(\|x\|)+\left\langle y, J_{\varphi}(x+y)\right\rangle .
$$

In particular, for all $x, y \in E$,

$$
\|x+y\|^{2} \leq\|x\|^{2}+2\langle y, J(x+y)\rangle .
$$

(ii) Assume that a sequence $\left\{x_{n}\right\}$ in E converges weakly to a point $x \in E$.

Then the following identity holds:

$$
\limsup _{n \rightarrow \infty} \Phi\left(\left\|x_{n}-y\right\|\right)=\limsup _{n \rightarrow \infty} \Phi\left(\left\|x_{n}-x\right\|\right)+\Phi(\|y-x\|), \quad \forall x, y \in E .
$$

LeMma 1.2 ([31]). Assume that $\left\{\alpha_{n}\right\}$ is a sequence of non-negative real numbers such that

$$
\alpha_{n+1} \leq\left(1-\gamma_{n}\right) \alpha_{n}+\delta_{n}, \quad \forall n \geq 1,
$$

where $\left\{\gamma_{n}\right\}$ is a sequence in $(0,1)$ and $\left\{\delta_{n}\right\}$ is a sequence such that

(i) $\sum_{n=1}^{\infty} \gamma_{n}=\infty$;

(ii) $\limsup _{n \rightarrow \infty} \frac{\delta_{n}}{\gamma_{n}} \leq 0$ or $\sum_{n=1}^{\infty}\left|\delta_{n}\right|<\infty$.

Then $\lim _{n \rightarrow \infty} \alpha_{n}=0$.

The following lemma can be deduced from Bruck [3] and Suzuki [26].

LeMmA 1.3. Let $C$ be a closed convex subset of a strictly convex Banach space E. Let $\left\{T_{n}\right\}_{n=1}^{N}$ be a sequence of non-expansive mappings on $C$. Suppose that $\cap_{n=1}^{N} F\left(T_{n}\right)$ is nonempty. Let $\left\{\lambda_{n}\right\}$ be a sequence of positive numbers with $\sum_{n=1}^{N} \lambda_{n}=1$. Then a mapping $S$ on $C$ defined by

$$
S x=\sum_{n=1}^{N} \lambda_{n} T_{n} x
$$

for $x \in C$ is well defined, non-expansive and $F(S)=\cap_{n=1}^{N} F\left(T_{n}\right)$ holds.

LEMMA 1.4 ([14]). Let $E$ be a Banach space satisfying a weakly continuous duality map, $C$ a non-empty closed convex subset of $E$ and $T: C \rightarrow C$ a non-expansive mapping with a fixed point. Then $I-T$ is demi-closed at zero, i.e., if $\left\{x_{n}\right\}$ is a sequence in $C$ which converges weakly to $x$ and if the sequence $\left\{(I-T) x_{n}\right\}$ converges strongly to zero, then $x=T x$.

\section{Main results.}

THEOREM 2.1. Let $C$ be a non-empty closed convex subset of a reflexive and strictly convex Banach space $E$ which also has a weakly continuous duality map $J_{\varphi}(x)$ with the gauge $\varphi$. Let $S$ and $T$ be non-expansive mappings from $C$ into itself such that $F=F(S) \cap F(T) \neq \emptyset$. Let $\left\{\alpha_{n}\right\}$ and $\left\{\beta_{n}\right\}$ be sequences in $(0,1)$. Let $\left\{x_{n}\right\}$ be a sequence 
defined by

$$
\left\{\begin{array}{l}
x_{0} \in C \\
y_{n}=\beta_{n} S x_{n}+\left(1-\beta_{n}\right) T x_{n}, \\
x_{n+1}=\alpha_{n} u+\left(1-\alpha_{n}\right) y_{n}, \quad n \geq 0
\end{array}\right.
$$

where $u \in C$ is a given point. Assume that the following restrictions imposed on the control sequences are satisfied:

(a) $\sum_{n=0}^{\infty} \alpha_{n}=\infty, \quad \lim _{n \rightarrow \infty} \alpha_{n}=0$;

(b) $\sum_{n=0}^{\infty}\left|\alpha_{n+1}-\alpha_{n}\right|<\infty, \quad \sum_{n=0}^{\infty}\left|\beta_{n+1}-\beta_{n}\right|<\infty$;

(c) $\lim _{n \rightarrow \infty} \beta_{n}=\beta \in(0,1)$.

Then the sequence $\left\{x_{n}\right\}$ generated by $(\Upsilon)$ converges strongly to $x^{*} \in F$, where $x^{*}=Q(u)$ and $Q: C \rightarrow F$ is the unique sunny non-expansive retraction from $C$ onto $F$.

Proof. First, we show that the sequences $\left\{x_{n}\right\}$ and $\left\{y_{n}\right\}$ are bounded. For any $p \in F$, we have

$$
\begin{aligned}
\left\|y_{n}-p\right\| & =\left\|\beta_{n} S x_{n}+\left(1-\beta_{n}\right) T x_{n}-p\right\| \\
& \leq \beta\left\|S x_{n}-p\right\|+\left(1-\beta_{n}\right)\left\|T x_{n}-p\right\| \\
& \leq \beta\left\|x_{n}-p\right\|+\left(1-\beta_{n}\right)\left\|x_{n}-p\right\| \\
& =\left\|x_{n}-p\right\| .
\end{aligned}
$$

It follows that

$$
\begin{aligned}
\left\|x_{n+1}-p\right\| & =\left\|\alpha_{n}(u-p)+\left(1-\alpha_{n}\right)\left(y_{n}-p\right)\right\| \\
& \leq \alpha_{n}\|u-p\|+\left(1-\alpha_{n}\right)\left\|y_{n}-p\right\| \\
& \leq \alpha_{n}\|u-p\|+\left(1-\alpha_{n}\right)\left\|x_{n}-p\right\| .
\end{aligned}
$$

By simple inductions, we have

$$
\left\|x_{n}-p\right\| \leq \max \left\{\left\|x_{0}-p\right\|,\|u-p\|\right\},
$$

which gives that the sequence $\left\{x_{n}\right\}$ is bounded, so is $\left\{y_{n}\right\}$.

From the iterative process $(\Upsilon)$, we have

$$
\begin{aligned}
y_{n}-y_{n-1}= & \beta_{n} S x_{n}+\left(1-\beta_{n}\right) T x_{n}-\left[\beta_{n-1} S x_{n-1}+\left(1-\beta_{n-1}\right) T x_{n-1}\right] \\
= & \beta_{n}\left(S x_{n}-S x_{n-1}\right)+S x_{n-1}\left(\beta_{n}-\beta_{n-1}\right)+\left(1-\beta_{n}\right)\left(T x_{n}-T x_{n-1}\right) \\
& +T x_{n-1}\left(\beta_{n-1}-\beta_{n}\right) .
\end{aligned}
$$

It follows that

$$
\begin{aligned}
& \left\|y_{n}-y_{n-1}\right\| \\
& \quad \leq \beta_{n}\left\|S x_{n}-S x_{n-1}\right\|+\left\|S x_{n-1}\right\|\left|\beta_{n}-\beta_{n-1}\right|+\left(1-\beta_{n}\right)\left\|T x_{n}-T x_{n-1}\right\| \\
& \quad+\left\|T x_{n-1}\right\|\left|\beta_{n-1}-\beta_{n}\right| \\
& \quad \leq \beta_{n}\left\|x_{n}-x_{n-1}\right\|+\left\|S x_{n-1}\right\|\left|\beta_{n}-\beta_{n-1}\right|+\left(1-\beta_{n}\right)\left\|x_{n}-x_{n-1}\right\| \\
& \quad+\left\|T x_{n-1}\right\|\left|\beta_{n-1}-\beta_{n}\right| \\
& \quad \leq\left\|x_{n}-x_{n-1}\right\|+M_{1}\left|\beta_{n}-\beta_{n-1}\right|
\end{aligned}
$$

where $M_{1}$ is an appropriate constant such that $M_{1} \geq \sup _{n \geq 0}\left\{\left\|S x_{n}\right\|+\left\|T x_{n}\right\|\right\}$. 
On the other hand, from the iterative process $(\Upsilon)$, we also have

$$
\begin{aligned}
x_{n+1}-x_{n} & =\alpha_{n} u+\left(1-\alpha_{n}\right) y_{n}-\left[\alpha_{n-1} u+\left(1-\alpha_{n-1}\right) y_{n-1}\right] \\
& =\left(\alpha_{n}-\alpha_{n-1}\right)\left(u-y_{n-1}\right)+\left(1-\alpha_{n}\right)\left(y_{n}-y_{n-1}\right)
\end{aligned}
$$

This implies that

$$
\left\|x_{n+1}-x_{n}\right\| \leq\left|\alpha_{n}-\alpha_{n-1}\right|\left\|u-y_{n-1}\right\|+\left(1-\alpha_{n}\right)\left\|y_{n}-y_{n-1}\right\| .
$$

Substituting (2.1) into (2.2), we arrive at

$$
\begin{aligned}
\left\|x_{n+1}-x_{n}\right\| & \leq\left|\alpha_{n}-\alpha_{n-1}\right|\left\|u-y_{n-1}\right\|+\left(1-\alpha_{n}\right)\left(\left\|x_{n}-x_{n-1}\right\|+M_{1}\left|\beta_{n}-\beta_{n-1}\right|\right) \\
& \leq\left(1-\alpha_{n}\right)\left\|x_{n}-x_{n-1}\right\|+M_{2}\left(\left|\beta_{n}-\beta_{n-1}\right|+\left|\alpha_{n}-\alpha_{n-1}\right|\right),
\end{aligned}
$$

where $M_{2}$ is an appropriate constant such that $M_{2} \geq \max \left\{\sup _{n \geq 0}\left\{\left\|u-y_{n}\right\|, M_{1}\right\}\right.$. From the conditions (a) and (b) and applying Lemma 1.2 to (2.3), we see that

$$
\lim _{n \rightarrow \infty}\left\|x_{n+1}-x_{n}\right\|=0 .
$$

Put $U=\beta S+(1-\beta) T$. From Lemma 1.3, we have that $U$ is non-expansive with $F=F(U)$. Notice that

$$
\begin{aligned}
x_{n}-U x_{n}= & x_{n}-x_{n+1}+x_{n+1}-U x_{n} \\
= & x_{n}-x_{n+1}+\alpha_{n} u+\left(1-\alpha_{n}\right)\left[\beta n S x_{n}+\left(1-\beta_{n}\right) T x_{n}\right]-U x_{n} \\
= & x_{n}-x_{n+1}+\alpha_{n}\left(u-U x_{n}\right)+\left(1-\alpha_{n}\right)\left[\beta_{n} S x_{n}+\left(1-\beta_{n}\right) T x_{n}-U x_{n}\right] \\
= & x_{n}-x_{n+1}+\alpha_{n}\left(u-U x_{n}\right) \\
& +\left(1-\alpha_{n}\right)\left[\beta_{n} S x_{n}+\left(1-\beta_{n}\right) T x_{n}-\beta S x_{n}-(1-\beta) T x_{n}\right] \\
= & x_{n}-x_{n+1}+\alpha_{n}\left(u-U x_{n}\right)+\left(1-\alpha_{n}\right)\left(\beta_{n}-\beta\right)\left(S x_{n}-T x_{n}\right)
\end{aligned}
$$

It follows that

$$
\left\|x_{n}-U x_{n}\right\| \leq\left\|x_{n}-x_{n+1}\right\|+\alpha_{n}\left\|u-U x_{n}\right\|+\left|\beta_{n}-\beta\right|\left\|S x_{n}-T x_{n}\right\| .
$$

It follows from the conditions (a), (c) and (2.4) that

$$
\lim _{n \rightarrow \infty}\left\|x_{n}-U x_{n}\right\|=0 .
$$

Next, we prove that

$$
\limsup _{n \rightarrow \infty}\left\langle u-Q(u), J_{\varphi}\left(x_{n}-Q(u)\right)\right\rangle \leq 0,
$$

where $Q$ is the sunny non-expansive retraction $Q: C \rightarrow F$. Take a subsequence $\left\{x_{n_{k}}\right\}$ of $\left\{x_{n}\right\}$ such that

$$
\limsup _{n \rightarrow \infty}\left\langle u-Q(u), J_{\varphi}\left(x_{n}-Q(u)\right\rangle=\lim _{k \rightarrow \infty}\left\langle u-Q(u), J_{\varphi}\left(x_{n_{k}}-Q(u)\right\rangle .\right.\right.
$$

Since $E$ is reflexive and the sequence $\left\{x_{n}\right\}$ is bounded, we may assume that $x_{n_{k}} \rightarrow \bar{x}$ for some $\bar{x} \in C$. From (2.5), we have that

$$
\lim _{n \rightarrow \infty}\left\|x_{n_{k}}-U x_{n_{k}}\right\|=0 .
$$


Thanks to Lemma 1.4, we see that

$$
\bar{x} \in F(U)=F(S) \cap F(T) .
$$

It follows from (2.7) that

$$
\limsup _{n \rightarrow \infty}\left\langle u-Q(u), J_{\varphi}\left(x_{n}-Q(u)\right)\right\rangle=\left\langle u-Q(u), J_{\varphi}(\bar{x}-Q(u))\right\rangle \leq 0 .
$$

Hence, we obtain that (2.6) holds.

Finally, we prove that $x_{n} \rightarrow Q(u)$ as $n \rightarrow \infty$. Notice that

$$
\begin{aligned}
\Phi\left(\left\|y_{n}-Q(u)\right\|\right) & =\Phi\left(\left\|\beta_{n}\left(S x_{n}-Q(u)\right)+\left(1-\beta_{n}\right)\left(T x_{n}-Q(u)\right)\right\|\right) \\
& \leq \Phi\left(\beta_{n} \|\left(S x_{n}-Q(u)\left\|+\left(1-\beta_{n}\right)\right\| T x_{n}-Q(u) \|\right)\right. \\
& \leq \Phi\left(\beta_{n} \|\left(x_{n}-Q(u)\left\|+\left(1-\beta_{n}\right)\right\| x_{n}-Q(u) \|\right)\right. \\
& \leq \Phi\left(\left\|x_{n}-Q(u)\right\|\right) .
\end{aligned}
$$

It follows that

$$
\begin{aligned}
\Phi\left(\left\|x_{n+1}-Q(u)\right\|\right) & =\Phi\left(\left\|\alpha_{n}(u-Q(u))+\left(1-\alpha_{n}\right)\left(y_{n}-Q(u)\right)\right\|\right) \\
& \leq \Phi\left(\left(1-\alpha_{n}\right)\left\|y_{n}-Q(u)\right\|\right)+\alpha_{n}\left\langle u-Q(u), J_{\varphi}\left(x_{n+1}-Q(u)\right)\right\rangle \\
& \leq\left(1-\alpha_{n}\right) \Phi\left(\left\|x_{n}-Q(u)\right\|\right)+\alpha_{n}\left\langle u-Q(u), J_{\varphi}\left(x_{n+1}-Q(u)\right)\right\rangle .
\end{aligned}
$$

From Lemma 1.2, we see that $\Phi\left(\left\|x_{n+1}-Q(u)\right\|\right) \rightarrow 0$ as $n \rightarrow \infty$, that is,

$$
\left\|x_{n}-Q(u)\right\| \rightarrow 0 \text { as } n \rightarrow \infty \text {. }
$$

This completes the proof.

Putting $S=I$, the identity mapping, we have the following.

COROLlARY 2.2. Let $C$ be a non-empty closed convex subset of a reflexive and strictly convex Banach space $E$ which also has a weakly continuous duality map $J_{\varphi}(x)$ with the gauge $\varphi$. Let $T$ be a non-expansive mapping from $C$ into itself such that $F(T) \neq \emptyset$. Let $\left\{\alpha_{n}\right\}$ and $\left\{\beta_{n}\right\}$ be sequences in $(0,1)$. Let $\left\{x_{n}\right\}$ be a sequence defined by the modified Mann iterative process (MMIP), where $u \in C$ is a given point. Assume that the following restrictions imposed on the control sequences are satisfied:

(a) $\sum_{n=0}^{\infty} \alpha_{n}=\infty, \quad \lim _{n \rightarrow \infty} \alpha_{n}=0$;

(b) $\sum_{n=0}^{\infty}\left|\alpha_{n+1}-\alpha_{n}\right|<\infty, \quad \sum_{n=0}^{\infty}\left|\beta_{n+1}-\beta_{n}\right|<\infty$;

(c) $\lim _{n \rightarrow \infty} \beta_{n}=\beta \in(0,1)$.

Then the sequence $\left\{x_{n}\right\}$ converges strongly to $x^{*} \in F(T)$, where $x^{*}=Q(u)$ and $Q: C \rightarrow$ $F(T)$ is the unique sunny non-expansive retraction from $C$ onto $F(T)$.

Putting $S=T$ in Theorem 2.1, we obtain the following.

COROLlARY 2.3. Let $C$ be a non-empty closed convex subset of a reflexive and strictly convex Banach space $E$ which also has a weakly continuous duality map $J_{\varphi}(x)$ with the gauge $\varphi$. Let $T$ be a non-expansive mapping from $C$ into itself such that $F(T) \neq \emptyset$. Let $\left\{\alpha_{n}\right\}$ and $\left\{\beta_{n}\right\}$ be sequences in $(0,1)$. Let $\left\{x_{n}\right\}$ be a sequence defined by the Halpern's iterative process (HIP), where $u \in C$ is a given point. Assume that the following restrictions imposed on the control sequences are satisfied: 
(a) $\sum_{n=0}^{\infty} \alpha_{n}=\infty, \quad \lim _{n \rightarrow \infty} \alpha_{n}=0$;

(b) $\sum_{n=0}^{\infty}\left|\alpha_{n+1}-\alpha_{n}\right|<\infty$.

Then the sequence $\left\{x_{n}\right\}$ converges strongly to $x^{*} \in F(t)$, where $x^{*}=Q(u)$ and $Q: C \rightarrow$ $F(T)$ is the unique sunny non-expansive retraction from $C$ onto $F(T)$.

3. Applications. Recently, many authors considered the following convex feasibility problem (CFP):

$$
\text { finding an } x \in \bigcap_{i=1}^{N} C_{i},
$$

where $N \geq 1$ is an integer and each $C_{i}$ is assumed to be the fixed point set of a nonexpansive mapping $T_{i}, i=1,2, \ldots, N$. There is a considerable investigation on CFP in the setting of Hilbert spaces which captures applications in various disciplines such as image restoration $[\mathbf{8}, \mathbf{1 3}]$, computer tomography [25] and radiation therapy treatment planning [4].

In this section, we study the CFP in the setting of Banach space. To be more precise, we introduce a parallel iterative algorithm for a finite family of non-expansive mappings in a real reflexive Banach space.

THEOREM 3.1. Let $C$ be a non-empty closed convex subset of a reflexive and strictly convex Banach space $E$ which also has a weakly continuous duality map $J_{\varphi}(x)$ with the gauge $\varphi$. Let $\left\{T_{i}\right\}_{i=1}^{N}$ be a finite family of non-expansive mappings from $C$ into itself such that $F=\cap_{i=1}^{N} F\left(T_{i}\right) \neq \emptyset$. Let $\left\{\alpha_{n}\right\}$ and $\left\{\beta_{n}^{i}\right\}$ be sequences in $(0,1)$. Let $\left\{x_{n}\right\}$ be a sequence defined by

$$
x_{0} \in C, \quad x_{n+1}=\alpha_{n} u+\left(1-\alpha_{n}\right) \sum_{i=1}^{N} \beta_{n}^{i} T_{i} x_{n}, \quad n \geq 0,
$$

where $u \in C$ is a given point. Assume that the following restrictions imposed on the control sequences are satisfied:

(a) $\sum_{n=0}^{\infty} \alpha_{n}=\infty, \quad \lim _{n \rightarrow \infty} \alpha_{n}=0$;

(b) $\sum_{n=0}^{\infty}\left|\alpha_{n+1}-\alpha_{n}\right|<\infty, \quad \sum_{n=0}^{\infty} \sum_{i=1}^{N}\left|\beta_{n+1}^{i}-\beta_{n}^{i}\right|<\infty$;

(c) $\lim _{n \rightarrow \infty} \beta_{n}^{i}=\beta^{i} \in(0,1)$ and $\sum_{i=1}^{N} \beta_{n}^{i}=1$.

Then the sequence $\left\{x_{n}\right\}$ generated by $\left(\Upsilon^{\prime}\right)$ converges strongly to $x^{*} \in F$, where $x^{*}=Q(u)$ and $Q: C \rightarrow F$ is the unique sunny non-expansive retraction from $C$ onto $F$.

Proof. From the proof of Theorem 2.1, we can conclude the desired conclusion easily.

REMARK 3.2. If $f: C \rightarrow C$ is a contractive mapping and we replace $u$ by $f\left(x_{n}\right)$ in the recursion formula $(\Upsilon)$, we can obtain the so-called viscosity iteration method. We note that all theorems and corollaries of this paper carry over trivially to the so-called viscosity iteration method, see [27] for more details.

\section{REFERENCES}

1. F. E. Browder, Fixed point theorems for noncompact mappings in Hilbert spaces, Proc. Natl. Acad. Sci. USA 53 (1965), 1272-1276.

2. F. E. Browder, Convergence theorems for sequences of nonlinear operators in Banach spaces, Math. Z. 100 (1967), 201-225. 
3. R. E. Bruck, Properties of fixed point sets of nonexpansive mappings in Banach spaces, Trans. Amer. Math. Soc. 179 (1973), 251-262.

4. Y. Censor and S. A. Zenios, Parallel Optimization. Theory, Algorithms, and Applications, Numerical Mathematics and Scientific Computation (Oxford University Press, New York, 1997).

5. Y. J. Cho, S. M. Kang and X. Qin, Approximation of common fixed points of an infinite family of nonexpansive mappings in Banach spaces, Comput. Math. Appl. 56 (2008), 20582064.

6. Y. J. Cho, S. M. Kang and X. Qin, Convergence theorems of fixed points for a finite family of nonexpansive mappings in Banach spaces, Fixed Point Theory Appl. 2008 (2008),1-6.

7. Y. J. Cho and X. Qin, Convergence of a general iterative method for nonexpansive mappings in Hilbert spaces, J. Comput. Appl. Math. 228 (2009), 458-465.

8. P. L. Combettes, The convex feasibility problem: In image recovery, Advances in Imaging and Electron Physics (P. Hawkes, Editor) (Academic Press, Orlando, FA, 1996), vol. 95, pp. 155270.

9. K. Goebel and S. Reich, Uniform convexity, hyperbolic geometry and nonexpansive mappings (Dekker, New York, 1984).

10. B. Halpern, Fixed points of nonexpanding maps, Bull. Amer. Math. Soc. 73 (1967), 957-961.

11. T. H. Kim and H. K. Xu, Strong convergence of modified Mann iterations, Nonlinear Anal. 61 (2005), 51-60.

12. Y. Kimura, W. Takahashi and M. Toyoda, Convergence to common fixed points of a finite family of nonexpansive mappigns, Arch. Math. 84 (2005), 350-363.

13. T. Kotzer, N. Cohen and J. Shamir, Images to ration by a novel method of parallel projection onto constraint sets, Opt. Lett. 20 (1995), 1172-1174.

14. T. C. Lim and H. K. Xu, Fixed point theorems for asymptotically nonexpansive mappings, Nonlinear Anal. 22 (1994), 1345-1355.

15. P. L. Lions, Approximation de points fixes de contractions, C.R. Acad. Sci. Sèr. A-B Paris 284 (1977), 1357-1359. 510.

16. W. R. Mann, Mean value methods in iteration, Proc. Amer. Math. Soc. 4 (1953), 506-

17. K. Nakajo and W. Takahashi, Strong convergence theorems for nonexpansive mappings and nonexpansive semigroups, J. Math. Anal. Appl. 279 (2003), 372-379.

18. X. Qin, Y. J. Cho, J. I. Kang and S. M. Kang, Strong convergence theorems for an infinite family of nonexpansive mappings in Banach spaces, J. Comput. Appl. Math. 230 (2009), $121-127$.

19. X. Qin and Y. Su, Approximation of a zero point of accretive operator in Banach spaces, J. Math. Anal. Appl. 329 (2007), 415-424.

20. X. Qin, Y. Su and M. Shang, Strong convergence of the composite Halpern iteration, J. Math. Anal. Appl. 339 (2008), 996-1002.

21. X. Qin, Y. Su and C. Wu, Strong convergence theorems for nonexpansive mappings by viscosity approximation methods in Banach spaces, Math. J. Okayama Univ. 50 (2008), 113-125.

22. S. Reich, Weak convergence theorems for nonexpansive mappings in Banach spaces, J. Math. Anal. Appl. 67 (1979), 274-276.

23. S. Reich, Strong convergence theorems for resolvents of accretive operators in Banach spaces, J. Math. Anal. Appl. 75 (1980), 287-292.

24. Y. Su and X. Qin, Strong convergence theorems for asymptotically nonexpansive mappings and asymptotically nonexpansive semigroups, Fixed Point Theory Appl. 2006 (2006), $1-11$.

25. M. I. Sezan and H. Stark, Application of convex projection theory to image recovery in tomograph and related areas, in Image Recovery: Theory and Application (H. Stark, Editor) (Academic Press, Orlando, FA, 1987) pp. 155-270.

26. T. Suzuki, Strong convergence theorem to common fixed points of two nonexpansive mappings in general Banach spaces, J. Nonlinear Convex Anal. 3 (2002), 381-391.

27. T. Suzuki, Moudafi's viscosity approximations with Meir-Keeler contractions, J. Math. Anal. Appl. 325 (2007), 342-352.

28. R. Wittmann, Approximation of fixed points of nonexpansive mappings, Arch. Math. 58 (1992), 486-491. 
29. H. K. Xu, Another control condition in an iterative method for nonexpansive mappings, Bull. Austral. Math. Soc. 65 (2002), 109-113.

30. H. K. Xu, Iterative algorithms for nonlinear operators, J. Lond. Math. Soc. 66 (2002), 240-256.

31. H. K. Xu, An iterative approach to quadratic optimization, J. Optim. Theory Appl. 116 (2003), 659-678.

32. $\mathrm{H}$. K. Xu, Strong convergence of an iterative method for nonexpansive and accretive opertors, J. Math. Anal. Appl. 314 (2006), 631-643.

33. Y. Yao, R. Chen and J. C. Yao, Strong convergence and certain control conditions for modified Mann iteration, Nonliner Anal. 68 (2008), 1687-1693.

34. H. Zhou, Convergence theorems for $\lambda$-strict pseudo-contractions in 2-uniformly smooth Banach spaces, Nonlinear Anal. 69 (2008), 3160-3173. 